\title{
Different Mechanisms of Renal Na-K-ATPase Regulation by Dopamine in the Proximal and Distal Nephron
}

\author{
Takeo Satoh, Masayuki Ominato, and Adrian I. Katz*
}

\begin{abstract}
We reported a novel intracellular mechanism of renal Na-K-ATPase regulation by dopamine (DA) in the rat cortical collecting duct (CCD), which involves stimulation of protein kinase A (PKA) and phospholipase $A_{2}\left(P L A_{2}\right)$. In the present experiments we determined whether this mechanism also operates in other nephron segments. In the medullary thick ascending limbs (MTAL), DA and other cAMP agonists inhibited Na-K-ATPase activity, an effect that was abolished by PKA inhibitor IP $_{20}$, but various protein kinase $\mathrm{C}$ (PKC) activators did not, analogous to our previous findings in CCD. In sharp contrast, DA inhibition on Na-K-ATPase in the proximal convoluted tubule (PCT) was reproduced by PKC agonists. These effects was blocked by PKC inhibitor staurosporine, but not by $\mathbf{I P}_{20}$. Mepacrine, a PLA 2 inhibitor, reversed the pump effect of all agents, and arachidonic acid (AA) produced a dose-dependent pump inhibition, in all three nephron segments.

We conclude that the intercellular mechanisms of Na-K-ATPase regulation by dopamine differ in the proximal and distal nephron, as they involve stimulation of PKA in MTAL and CCD, and of PKC in PCT. These two pathways probably share a common mechanism in stimulating PLA $A_{2}$ and $A A$ release in both regions of the nephron. (Hypertens Res 1995; 18 Suppl. I: S137-S140)
\end{abstract}

Key Words: renal tubules, Na-K-ATPase, dopamine

Dopamine-induced natriuresis is attributed in part to inhibition of Na-K-ATPase activity, which has been demonstrated in the proximal convoluted tubule (PCT) (1), medullary thick ascending limb (MTAL) (2), and cortical collecting duct (CCD) (1). We recently reported a novel intracellular mechanism of Na-K-ATPase regulation in rat CCD that involves increased cell cAMP content, activation of protein kinase A (PKA) and phospholipase $\mathrm{A}_{2}\left(\mathrm{PLA}_{2}\right)$, and consequent increase in arachidonic acid production $(3,4)$. Available evidence suggest, however, that the intracellular signaling involved in Na-K-ATPase regulation by DA might differ in the proximal and distal nephron. In PCT activation of both $\mathrm{DA}_{1}$ and $\mathrm{DA}_{2}$ receptor is required for pump inhibition, whereas in MTAL and CCD activation of the $\mathrm{DA}_{1}$ receptor alone is sufficient to inhibit Na$\mathrm{K}$-ATPase activity (5). Since DA is reported to stimulate both PKA and protein kinase C (PKC) $(6)$, the pump inhibition by DA may be mediated either or both of these two kinases. We therefore undertook the present study to clarify the intracellular signaling pathways involved in Na-K-ATPase regulation along the nephron by dopamine using microdissected rat tubules.

\section{Methods}

All experiments were performed in vitro on freshly microdissected rat tubules. Incubation with or without agonists were carried out at room temperature for PCT or $37^{\circ} \mathrm{C}$ for MTAL and CCD. Na-KATPase activity was measured in single pieces of permeabilized tubules in Hanks' medium containing $\left[\gamma-{ }^{32} \mathrm{P}\right]$ ATP in the presence or absence of $4 \mathrm{mM}$ ouabain. Details of the microdissection and Na-KATPase microassay methods utilized have been described previously (7).

\section{Results and Discussion}

DA and a $\mathrm{DA}_{1}$ agonist fenoldopam (both $10^{-5} \mathrm{M}$ ) inhibited the Na-K-ATPase activity in CCD and MTAL, effects reproduced by forskolin $\left(10^{-5} \mathrm{M}\right)$ or dBcAMP $\left(10^{-3} \mathrm{M}\right)$ (Fig. 1, upper and middle panels). In contrast, only DA among these compound significantly inhibited the pump in PCT (not shown). We next examined the effect of PKC activators on Na-K-ATPase activity in individual nephron segments. PKC activators phorbol dibutyrate, phorbol myristate acetate (both, $10^{-6} \mathrm{M}$ ) or DiC8 $\left(10^{-4} \mathrm{M}\right)$ inhibited the pump activity in PCT

From the First Department of Internal Medicine, St. Mariannna University School of Medicine, Kawasaki, Japan, * the Department of Medicine, University of Chicago Pritzker School of Medicine, Chicago, USA.

Address for Reprints: Takeo Satoh, M.D., First Department of Internal Medicine, St. Mariannna University School of Medicine, 2-16-1 Sugao, Miyamae-ku, Kawasaki, 216, Japan. 


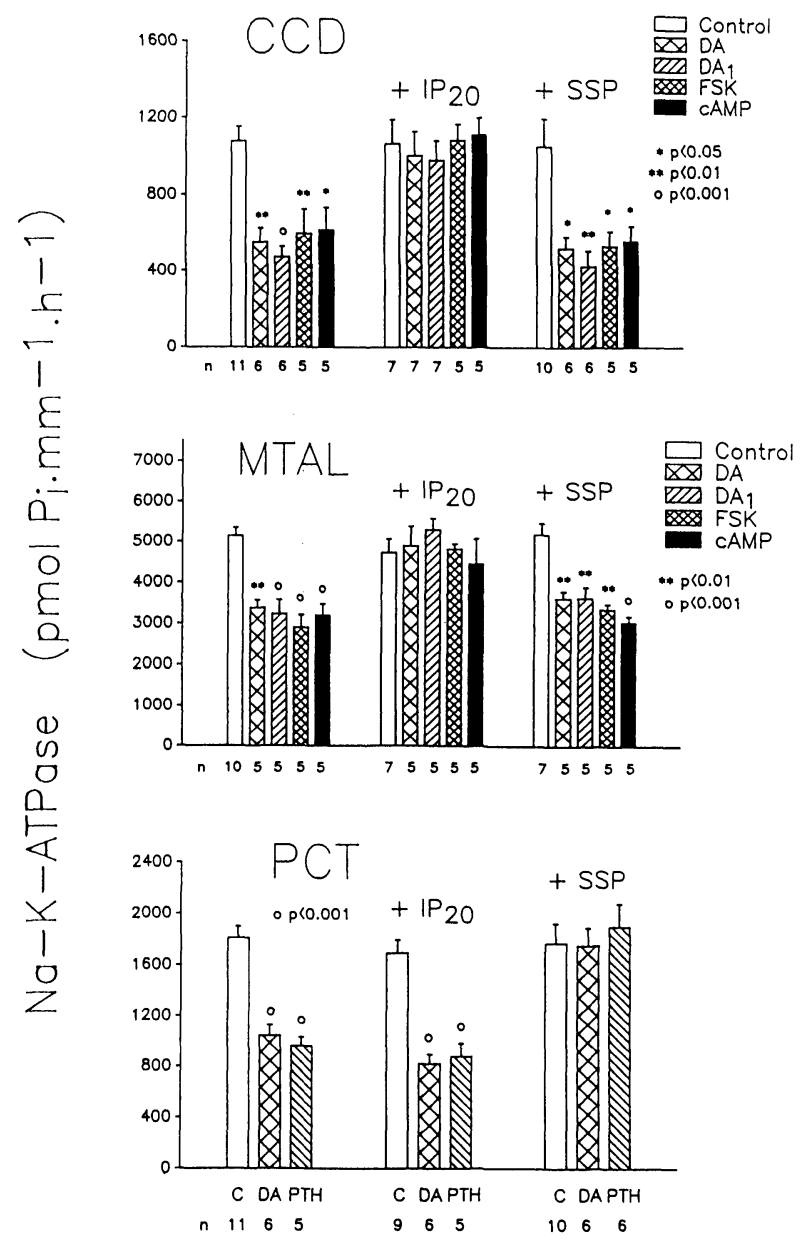

Fig. 1. Inhibition of PKA (IP20) but not PKC (SSP) abolishes the effect of $c A M P$ agonists on Na-K-ATPase activity in the cortical collecting duct (upper panel) and medullary thick ascending limb (middle panel). In contrast, SSP but not IP 20 blocks the effects of dopamine or $P T H$ on Na-K-ATPase activity in the proximal convoluted tubule (lower panel).

$I P_{20}, 20$ residue $P K A$ inhibitory peptide; SSP, staurosporine.

but not in MTAL and CCD (not shown), results opposite to those with cAMP agonists described above. $\operatorname{IP}_{20}\left(10^{-8} \mathrm{M}\right)$, a peptide inhibitor of PKA, abolished the pump inhibition by DA, fenoldopam, forskolin or dBcAMP in CCD and MTAL. In contrast, staurosporine $\left(10^{-7} \mathrm{M}\right)$ had no effect on Na$\mathrm{K}$-ATPase inhibition by these agents in either nephron segment (Fig. 1, upper and middle panels). Pump inhibition by DA or PTH in PCT, on the other hand, was completely prevented by staurosporine but not by $\mathrm{IP}_{20}$ (Fig. 1, lower panel). Moreover, DA or PTH effect was prevented by U73122, an inhibitor of phospholipase C (PLC) (not shown). These observations strongly suggest that in MTAL and CCD Na-K-ATPase inhibition by DA is mediated by adenylate cyclase-PKA pathway, whereas in PCT, DA action on the pump is mediated by the PLC-PKC pathway.

PKA stimulates phospholipase $\mathrm{A}_{2}\left(\mathrm{PLA}_{2}\right)(8)$,

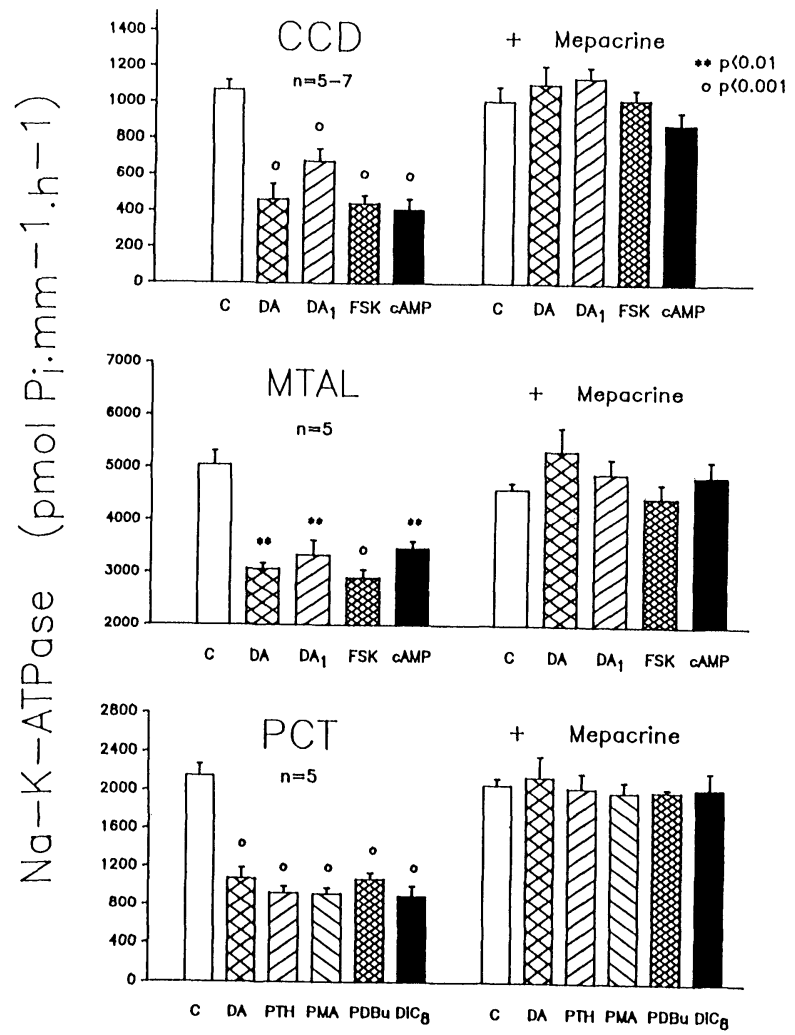

Fig. 2. Mepacrine reverses Na-K-ATPase inhibition by cAMP-PKA agonists in the CCD and MTAL, and by $P K C$ agonists in the PCT.

leading to increased arachidonic acid release and production of arachidonate metabolites instrumental in Na-K-ATPase inhibition in CCD. Because PKC also stimulates $\mathrm{PLA}_{2}$ (9), we evaluated whether $\mathrm{PLA}_{2}$ activation also participate in Na-K-ATPase regulation in the proximal nephron as well. Mepacrine $\left(10^{-5} \mathrm{M}\right)$, an inhibitor of PLA , blocked the inhibitory effect of DA and other cAMP agonists on Na-K-ATPase in CCD and MTAL, and did so that of DA and PTH, as well as of the three PKC agonists in PCT mentioned above (Fig. 2), suggesting that $\mathrm{PLA}_{2}$ stimulation is also involved in the signaling cascade in the PCT as well as in the distal nephron. This conclusion is further supported by results of studies with arachidonic acid, which caused dose-dependent inhibition of Na-K-ATPase activity in each nephron segments (not shown).

Our proposed scheme of intracellular signaling involved in the renal Na-K-ATPase regulation are summarized in Fig. 3. We propose that the renal Na-K-ATPase modulation by DA is mediated by different protein kinase in the proximal and distal nephron: whereas PLC-PKC pathway mediates the pump inhibition by DA in PCT, adenylate cyclasePKA does so in MTAL and CCD. These two pathways link to the activation of $\mathrm{PLA}_{2}$ and subsequent release of arachidonic acid, whose metabolites probably play key roles in the regulation of $\mathrm{Na}-\mathrm{K}-$ ATPase in each of these nephron segments. 


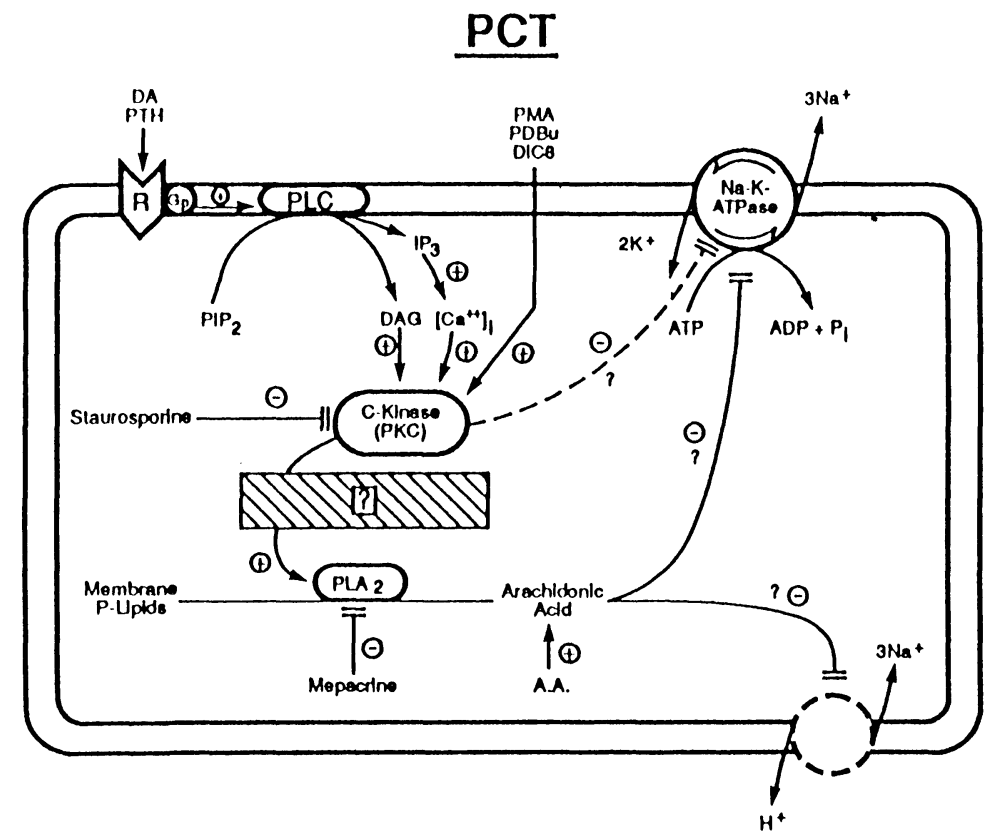

\section{CCD and MTAL}

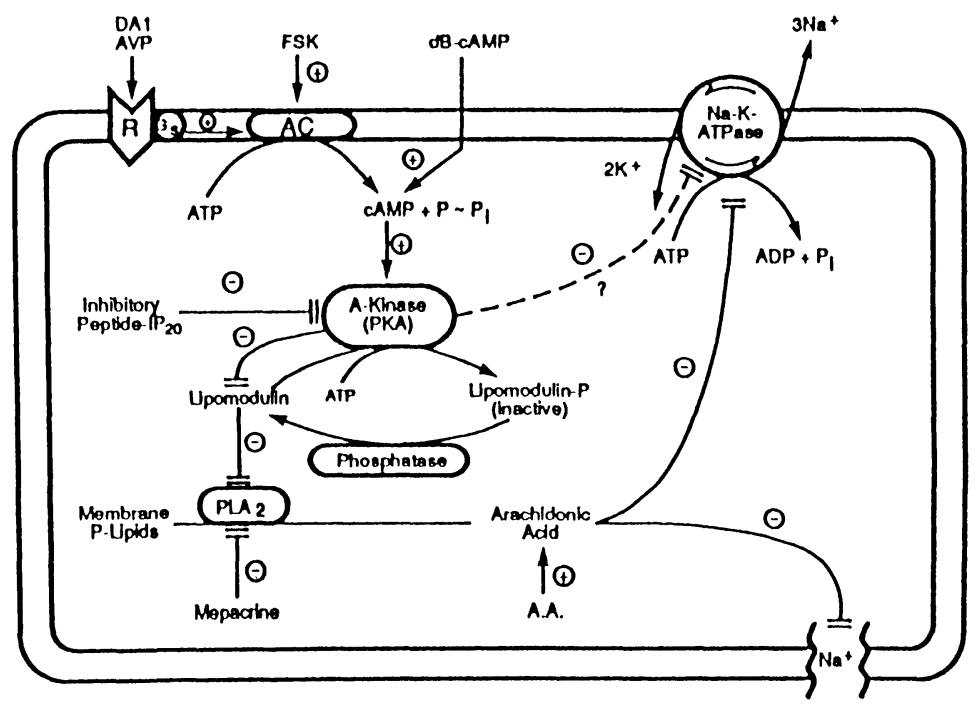

Fig. 3. Schematic representation of the proposed signaling pathways involved in Na-K-ATPase regulation in the PCT, and in the MTAL and CCD. Arrows with + signify stimulation; double lines with -, inhibition; and broken lines, possible direct phosphorylation by protein kinases.

\section{References}

1. Takemoto F, Satoh T, Cohen HT, Katz AI: Localization of dopamine-1 receptors along the microdissected rat nephron. Pflugers Arch 1991; 419: 243-248.

2. Meister B, Fryckstedt J, Schalling M, et al : Dopamine- and cAMP-regulated phosphoprotein (DARPP-32) and dopamine $\mathrm{DA}_{1}$ agonist-sensitive $\mathrm{Na}^{+}, \mathrm{K}^{+}$-ATPase in renal tubule cells. Proc Natl Acad Sci USA 1989; 86: 8068-8072.

3. Satoh T, Cohen HT, Katz AI: Intracellular signaling in the regulation of renal Na-K-ATPase: I. Role of cyclic AMP and phospholipase $\mathrm{A}_{2} . J$ Clin Invest 1992; 89: 1496-1500.

4. Satoh T, Cohen HT, Katz AI: Intracellular signaling in the regulation of renal Na-K-ATPase: II. Role of eicosanoids. J Clin Invest 1993; 91: 409-415.

5. Bertorello A, Aperia A: Inhibition of proximal tubule $\mathrm{Na}^{+}-\mathrm{K}^{+}$-ATPase activity requires simultaneous activation of $\mathrm{DA}_{1}$ and $\mathrm{DA}_{2}$ receptors. $A m J$ Physiol 1990; 259: F924-F928.

6. Felder CC, Blecher M, Jose PA: Dopamine-1-mediated stimulation of phospholipase $\mathrm{C}$ activity in rat renal cortical membranes. J Biol Chem 1989; 264: 
8739-8745.

7. Katz AI, Doucet A, Morel F: Na-K-ATPase activity along the rabbit, rat, and mouse nephron. Am J Physiol 1979; 237: F114-F120.

8. Hirata F: The regulation of lipomodulin, a phosholipase inhibitory protein, in rabbit neutrophils by phos- phorylation. J Biol Chem 1981; 256: 7730-7733.

9. Parker J, Daniel LW, Waite M: Evidence of protein kinase $\mathrm{C}$ involvement in phorbol diester-stimulated arachidonic acid release and prostaglandin synthesis. J Biol Chem 1991; 262: 5385-5393. 